\title{
Current diagnosis and treatment of cryptococcal meningitis without acquired immunodeficiency syndrome
}

\author{
Xiao-Su Guo, Hui Bu, Jun-Ying He, Yue-Li Zou, Yue Zhao, Yuan-Yuan Li, Jun-Zhao Cui, Ming-Ming Zheng, \\ Wei-Xin Han, Ze-Yan Zhao \\ Department of Neurology, the Second Hospital of Hebei Medical University, Shijiazhuang 050000, Hebei, China.
}

Correspondence to: Dr. Hui Bu, the Second Hospital of Hebei Medical University, 215 Heping West Road, Shijiazhuang 050000, Hebei, China. E-mail: buhuimy1@163.com

How to cite this article: Guo XS, Bu H, He JY, Zou YL, Zhao Y, Li YY, Cui JZ, Zheng MM, Han WX, Zhao ZY. Current diagnosis and treatment of cryptococcal meningitis without acquired immunodeficiency syndrome. Neuroimmunol Neuroinflammation 2016;3:249-56.

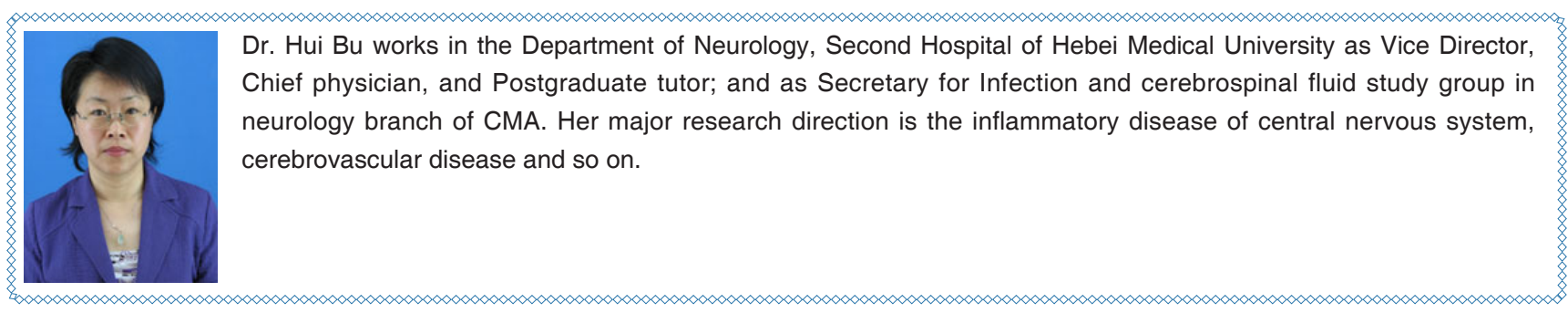

Article history:

Received: 19-02-2016

Accepted: 02-11-2016

Published: 18-11-2016

Key words:

Cryptococcal meningitis,

without AIDS,

diagnosis,

treatment

\begin{abstract}
Cryptococcal meningitis (CM) is a central nervous system infectious disease caused by Cryptococcus. It is the most common fungal infection in the central nervous system, accounting for about $48 \%$ of fungal infection. The disease occurs mainly in acquired immunodeficiency syndrome (AIDS) patients and concentrates in the immunocompromised people without AIDS. There are nearly one million new cases of CM each year, and about $70 \%$ of them died. In China, $\mathrm{CM}$ occurs mainly in people without AIDS and there is an increasing trend in recent years. Early diagnosis and treatment is the key to reducing morbidity and mortality associated with CM. The diagnosis mainly depends on laboratory examination such as morphological examination, fungal culture and antigen detection. History, clinical manifestation and imaging examination are the important parts of auxiliary examination. The initial combined antifungal treatment is emphasized, and the principle of fractional treatment including induction, consolidation and maintenance therapy should be followed. The high intracranial pressure must be reduced actively at the same time. In addition, it is proved that the novel immunotherapy combined with antifungal agents can improve the curative effect and limit the chance of antimicrobial resistance. Large-scale clinical trials are needed for further study.
\end{abstract}




\section{INTRODUCTION}

Cryptococcal meningitis (CM) is the most common cause of fungal meningitis worldwide. Globally, there are approximately 957,900 new cases of CM each year, and about 624,700 of them died. ${ }^{[1]}$ CM occurs mainly in the acquired immunodeficiency syndrome (AIDS) crowd abroad. In China, CM is sporadic, mainly in people without AIDS. In recent years, there is an increasing trend for the incidence of $\mathrm{CM}$ as a result of the wide application of antibiotic, hormone and immune inhibitors, organ transplantation in China. In developing countries, up to $70 \%$ of $\mathrm{CM}$ lead to death eventually. ${ }^{[2]}$ The severity of disease and limited access to diagnostics and medications results in the high mortality of $\mathrm{CM}$ in resource-limited settings (RLS). ${ }^{[1]}$ Early diagnosis and treatment is the key to reduce the morbidity and mortality.

No symptoms, hardship to select pathogen or lack of awareness in the early stages of the disease make the diagnosis difficult, particularly in RLS. The clinical manifestations and part of cerebrospinal fluid parameters such as fever, headache, high intracranial pressure, high protein and low glucose in cerebrospinal fluid (CSF) which are easily confused with tuberculous meningitis. Substantial resources, such as hospitalization, intravenous antifungal therapy, access to lumbar punctures, and strict monitoring are required in the process of $\mathrm{CM}$ treatment. ${ }^{[3]}$ In this review, we mainly describe the available diagnostic methods and management of CM without AIDS.

\section{DIAGNOSE OF CRYPTOCOCCAL MENINGITIS}

The diagnosis of the $\mathrm{CM}$ is dependent on the medical history, clinical manifestations, imageological examination, cerebrospinal fluid parameters and laboratory tests. Among them, laboratory tests are the main methods to make a definite diagnosis. India ink staining and fungal cultures are regarded as the diagnostic gold standard. There is not much difference in diagnostic criteria of $\mathrm{CM}$ between patients with or without human immunodeficiency virus (HIV). Merely for patients with advanced HIV, World Health Organization (WHO) recomends early cryptococcal antigen (CrAg) screening and treatment in 2011. ${ }^{[4]}$

\section{Medical history and clinical manifestation}

The medical history of $\mathrm{CM}$ includes environment (the contact history of pigeons) and susceptible population with risk factors including long term treatment of immunosuppressant, broad-spectrum antibiotics and glucocorticoids, HIV infection and patients with immunodeficiency is important for providing initial clues and diagnostic evidence. The CM has a hidden onset and a slow course, mainly presenting the symptoms of increased intracranial pressure (headache, nausea, vomiting, and disturbance of consciousness), meningeal irritation sign (neck rigidity, Kernig sign and Brudzinski sign). Patients with altered mental status have high mortality. ${ }^{[5-7]}$

Patients with typical symptoms of the meningeal irritation are less than $20 \%{ }^{[6]}$ Optic nerve damage is the most common among injury of cranial nerves caused by intracranial hypertension (optic nerve, oculomotor nerve, abducens nerve, facial nerve, vestibulocochlear nerve involvement). Forty percent of the patients with $\mathrm{CM}$ have visual involvement, including optic discedema and uveitis. ${ }^{[8-10]}$ Second is the vestibulocochlear nerve damage. If there is parenchymal involvement, it would appear epilepsy seizures, hemiplegia, mental disorder, ataxia, etc.

\section{Imaging examination}

Computed tomography (CT) and magnetic resonance imaging (MRI) has limited effect on the diagnosis, but it is necessary to find the complications (intracranial mass and hydrocephalus). ${ }^{[11]}$ Some professors divide the $\mathrm{CM}$ course into three periods. ${ }^{[12]}$ Acute phase: cerebral edema is showed on CT or MRI. Brain parenchyma presents punctate low-density lesions and Long T1, long T2 signal area, it is similar to cerebral infarction, called "soap bubble damage" ${ }^{\text {[12,13] }}$ which is caused by the expansion of the space (Robin Virchow) around the capillary. Subacute stage: Multifocal gelatinous pseudocysts formed in the deep white matter on both sides of the cerebral hemispheres, basal ganglia, thalamus and midbrain, etc. Chronic phase: intracranial single or multiple rounds, oval and sheet, etc., slightly higher or low density massive umbra, lesions surrounded by edema, may have mutual integration. Enhanced scan shows multiple small nodules ring, it is easy to be misdiagnosed as cerebral metastasis. Because of the correlation between CT/MRI and the disease progression or cerebrospinal fluid pressure, CT and MRI should be reviewed even if it was normal during the acute phase.

\section{CSF parameter}

The typical characteristic of cerebrospinal fluid for $\mathrm{CM}$ is high intracranial pressure (HICP) which is more than $350 \mathrm{mmH}_{2} \mathrm{O}$ or up to more than $900 \mathrm{mmH}_{2} \mathrm{O}$. The reason for HICP is that Cryptococcus hinder the CSF to pass through the arachnoid villi which obstructs the CSF circulation channel. ${ }^{[14]}$ Furthermore, the accumulation of capsular polysaccharide in arachnoid villi and subarachnoid spaces contributes to fluid retention by increasing the osmolarity of the CSF and 
interstitial fluid. ${ }^{[15,16]}$ The appearance of CSF is clear and transparent generally, and it can be slightly turbid if there is a large amount of Cryptococcus. Leukocyte count in CSF is increased (about 100-500 $\times 10^{6} / \mathrm{L}$ ) in the majority of people, or normal in the minority. In addition, the protein level rises (no more than $2 \mathrm{~g} / \mathrm{L}$ usually), and the glucose and chloride decreases in CSF as the result of infection. The degree of decrease in glucose levels is significantly lower than that of other central nervous system infection. ${ }^{[17]}$

The characteristics of cerebrospinal fluid cytology: the total number of cells increases to different degrees, presenting mixed cell reaction or lymphocyte dominated mixed cell reaction. Monocyte constitutes the main ingredients in the most of the cerebrospinal fluid cytology of CM patients, the total number of cells decreases and the proportion of small lymphocytes also increases with the improvement of the disease. Therefore, cerebrospinal fluid cytology has also certain significance to the monitoring of the efficacy.

However, cerebrospinal fluid examination is normal in $10-17 \%$ other patients, especially in the patients with HIV. ${ }^{[18,19]}$

\section{Other laboratory examination}

The common laboratory examination for diagnosing of CM mainly includes morphological examination, fungal culture, and antigen detection.

Cryptococcus neoformans (C. neoformans) is a singlecelled organism with a polysaccharide capsule. It exists in the blood, CSF, and tissues. ${ }^{[20]}$ Morphological diagnosis depends on dyeing technology. India ink staining is considered to be one of the gold standards for diagnosis. It is a traditional method of identification of $C$. neoformans, especially in areas with limited resources because of its simple and rapid operation. Characteristic "starry night" phenomenon ${ }^{[20]}$ would be observed by India ink staining: capsule is not shaded but surrounded dyed blue. Yet the sensitivity of india ink staining is only $<86 \%{ }^{[21,22]}$ In addition, due to low fungal loads, India ink is insensitive for patients who presenting early after symptoms appear or being on initiating antiretroviral therapy (ART). ${ }^{[23]}$ The detection rate of $C$. neoformans in the CSF is only $66 \%$ at first time, about $17.8 \%$ at second time, and others remain 3 to 20 times smear test under microscope to find positive. ${ }^{[24]}$ May-Grunwald-Giemsa (MGG) staining has a relatively high positive detection rate, a small amount of $C$. neoformans can be detected after centrifugal precipitation, applying to patients with low amount of fungi. But the morphological characteristics of the fungi are not clear, it is easy to be confused with small lymph cells in CSF when the $C$. neoformans scattered in the distribution. Therefore, the detection method requires high skill levels from observers. Alcian blue staining is a special dye for the C. neoformans ${ }^{[25]}$ and could dye the capsule to be deep blue, and the cell light blue, without the peripheral inflammatory cells being dyed. Therefore, the sensitivity of alcain blue staining is high and the fungi can be easily observed. ${ }^{[26]}$ The combination of the above methods can improve the detection rate. Culture is considered the gold standard for diagnosis of cryptococcal meningitis. ${ }^{[2]}$ But it is limited by culture conditions, culture time and the amount of cerebrospinal fluid and fungi, thus making the early diagnosis hampered. But it has important value for further drug sensitivity test and species classification.

The most reliable diagnostic method for cryptococcosis is to detect capsular polysaccharide antigen ${ }^{[20]}$ which can be find in serum, CSF, and urine specimens. Serum $\mathrm{CrAg}$ is taken as an early biologic marker which is far more sensitive and rapid than direct detection of the pathogen, it highly predicts of the development of $\mathrm{CM}$ within one year. ${ }^{[28]}$ Retrospective data suggests that CrAg screening in patients with late-stage human HIV ART may reduce cryptococcal disease and deaths. ${ }^{[29]}$ However, presence of CrAg in CSF is more valuable for diagnosis of $\mathrm{CM}$ and serum $\mathrm{CrAg}$ assay can help to assist the diagnosis.

Main methods are consist of latex agglutination (LA) assays, enzyme immunoassays (EIAs), or the novel lateral flow assay (LFA) ${ }^{[20]}$ LA or EIAs has been used for detecting $\mathrm{CrAg}$ for several years. ${ }^{[30]}$ The sensitivity and specificity of the LA test for CSF is high. The sensitivity ranges from $93 \%$ to $100 \%$, while the specificity ranges from $93 \%$ to $98 \%$, which is significantly better than India ink staining and CSF cytology in the early diagnosis of $\mathrm{CM}$. And the severity of the disease in patients with cryptococcal meningitis is correlated with the antigen titer of capsular polysaccharide, therefore, LA test also has the value of evaluating the severity of illness and the prognosis. ${ }^{[31]}$ Although this method has high sensitivity, but may appear false positive results in patients with immunological diseases, such as rheumatoid. ${ }^{[2,33]}$ Tedious manual operation and subjective intervention is the main weakness, in addition, this method needs equipment and refrigeration which restricts its application in resource limited area. ${ }^{[20]}$ The sensitivity and specificity of EIAs test for capsular polysaccharide antigen is high which is $100 \%$ and $98 \%$ respectively for CSF samples. ${ }^{[3,35]}$ However, EIAs test cannot be widely used because of the expensive detection kits.

The lateral flow assay (LFA) is developed in 2009, it could detect cryptococcal polysaccharide capsule 
quickly by using gold-conjugated anti-cryptococcal monoclonal antibodies directed at $C$. neoformans. ${ }^{[30]}$ It has higher sensitivity than LA and EIAs, and it is more sensitive for detecting the lower antigen in CSF. ${ }^{[22,30]}$ The CrAg LFA which is low cost can be carried out at room temperature without refrigeration or complicate experimental equipment, takes just $10 \mathrm{~min}$ to get the results. Therefore, it is expected to reform the diagnosis of cryptcoccosis in the restricted area. ${ }^{[36]}$

Recently, molecular biological detection comprised of chromosome pulse electrophoresis, nucleic acid probe, DNA fingerprinting technique and polymerase chain reaction (PCR) has been carried out in some laboratories. PCR are often used at present. PCR which applicates specific primer aimed at conservative sequence of $C$. neoformans to the detection of fungi is rapidly and pecifically. ${ }^{[31]}$ The primer used for multilocus sequence typing of $C$. neoformans includes CAP59, GPD1, IGS1, LAC1, PLB1, SOD1, URA5. ${ }^{[37]}$ The pathogenic fungi are identified as $C$. neoformans var. grubii, C. neoformans var. neoformans and hybrid strains by PCR. However, the requirements for the experimental technique of PCR is so high that this kind of test is not widely carried out in clinical practice at present. $^{[31]}$

\section{TREATMENT OF CRYPTOCOCCAL MENINGITIS}

Cryptococcal meningitis without treatment is fatal in most cases. It is critical to diagnose early and treat promptly for the improvement of survival. ${ }^{[38]}$

\section{Antifungal agents therapy}

Antifungal drugs used commonly include amphotericin B (AmB), 5-Flurocytosine (5-FC) and fluconazole (FCZ).

$A m B$ is a broad-spectrum antifungal agent. The mechanism of AmB is to combine with fungal cells membrane of ergosterol and interfere with cell metabolism and increase the cell membrane permeability aimed to bring about cell death. AmB is the first choice for the treatment of $\mathrm{CM}$, and it has the best early fungicidal activity (EFA).

$5-\mathrm{FC}$ is a pyrimidine analogues, and its mechanism of action is to inhibit cell division by interfering with the synthesis of fungal DNA. Single drug treatment is easy to produce drug resistance. $5-\mathrm{FC}$ is usually used incombination with $A m B$ and is superior to the combination of $\mathrm{AmB}$ and $\mathrm{FCZ} .{ }^{\left[{ }^{39]}\right.}$ The reason is that $\mathrm{AmB}$ has the ability to make the cell membrane permeability to increase, thus $5-\mathrm{FC}$ is more susceptible to enter the fungus and appear synergistic fungicidal effect. Without use of 5-FC in induction therapy will lead to increased mortality, treatment failure ${ }^{[40]}$ or recurrence..$^{[41]}$

FCZ is one of the triazole antifungal agents and its mechanism of action is to destroy the cell membrane and promotes cell death by inhibiting the activity of cytochrome P450 by inhibiting the synthesis of ergosterol in fungal cell membrane. ${ }^{[42]} \mathrm{FCZ}$ is easy to go through the blood brain barrier (BBB) to reach a high concentration in CSF. However, it belongs to fungistat that the effect of killing Cryptococcus is weaker than that of $\mathrm{AmB}$. Therefore, it can be used for sequential therapy after induction therapy. New drugs such as voriconazole and posaconazole have obvious anti-Cryptococcus activity in vitro.

Fractional treatment of the $\mathrm{CM}$ is recommended at present, consists of AmB plus 5-FC induction therapy, FCZ consolidation and maintenance therapy. ${ }^{[43]}$

Expert consensus of the diagnose and treatment of cryptococcal infection in China recommended combination therapy with AmB $0.5-1 \mathrm{mg} / \mathrm{kg}$ per day and $5-\mathrm{FC} 100 \mathrm{mg} / \mathrm{kg}$ per day as induction treatment for non-HIV associated patients which earned widespread approval from experts. ${ }^{[4]}$ The induction phase lasts at least 8 weeks which is different from the project of Infectious Diseases Society of America (IDSA), and this may be related to the use of the method in our country, namely it takes a period of time to begin with small dose to effective maintenance dose gradually. However, large-scale clinical trials are needed to demonstrate their validity. Then followed by consolidation therapy with FCZ or itraconazole $200-400 \mathrm{mg} /$ day at least 12 weeks, ${ }^{[24]}$ and maintenance therapy has not been mentioned in the Consensus [Table 1].

At present, there is a difference in the treatment of non-HIV associated patients, and its management is based on the characteristics other host and the pathogen. As a result of about $25 \%$ of the transplant patients are with renal dysfunction in the diagnosis of cryptococcal meningitis, ${ }^{[11]}$ liposome amphotericin B

Table 1: Antifungal therapeutic schedule for non-HIV associated CM patients

\begin{tabular}{lll}
\hline Schedule & Course & \\
\hline Induction period & AmB 0.5-1 mg/kg per day +5-FC $100 \mathrm{mg} / \mathrm{kg}$ per day & $\geq 8$ weeks \\
Consolidation period & FCZ/Itraconazole 200-400 mg/day & $\geq 12$ weeks \\
Maintenance period & Not mentioned & \\
\hline
\end{tabular}

HIV: human immunodeficiency virus; CM: cryptococcal meningitis; AmB: amphotericin B; 5-FC: 5-Flurocytosine; FCZ: fluconazole 
(LAmB)/amphotericin B lipid complexes (ABLC) with small renal toxicity are recommended in the induction period. ${ }^{[11,24]}$ In 2010 , IDSA ${ }^{[24]}$ suggests induction therapy with LAmB (3-4 mg/kg per day i.v.) or ABLC ( $5 \mathrm{mg} / \mathrm{kg}$ per day i.v.) plus $5-\mathrm{FC}(100 \mathrm{mg} / \mathrm{kg}$ per day i.v.) for at least 2 weeks, consolidation therapy with FCZ $400-800 \mathrm{mg}$ $(6-12 \mathrm{mg} / \mathrm{kg})$ per day for 8 weeks. And maintenance therapy with FCZ $200-400 \mathrm{mg}$ (3-6 mg/kg) per day lasts for 6-12 months [Table 2]. LAmB should be used at least 4-6 weeks without the use of 5-FC in induction therapy. Increasing dosage (6 $\mathrm{mg} / \mathrm{kg}$ per day) should be conducted when the fungal load is higher or palindromia.

Treatment for non-HIV associated or non-transplant patients includes induction therapy with $A m B$ 0.7-1.0 mg/kg per day or LAmB 3-4 mg/kg per day or ABLC $5 \mathrm{mg} / \mathrm{kg}$ per day plus $5-F C 100 \mathrm{mg} / \mathrm{kg}$ per day for 4-6 weeks. IDSA also recommends that it is essential to extend induction period if treated with $A m B / L A m B$ monotherapy or treatment interrupted. In addition, consolidation therapy with FCZ 400-800 mg (6-12 mg/kg) per day lasts for 8 weeks, and maintenance therapy with FCZ $200 \mathrm{mg}$ (3 $\mathrm{mg} / \mathrm{kg}$ ) per day lasts for 6-12 months [Table 3].

It is difficult to achieve effective concentration in the CSF for AmB or LAmB because of their poor ability to traverse BBB. Intravenous combined intrathecal administration of $\mathrm{AmB}$ can improve the drug concentration in CSF to inhibit the C. neoformans effectively, and observational studies suggest that it could be associated with improved survival. ${ }^{[45]}$ However, it is necessary to prevent the occurrence of complications caused by intrathecal administration , such as paresthesias, radiculitis, or myelopathy. ${ }^{[46]}$

Previous studies in humans and animals indicate that intrathecal administration of lipid formulations of AmB is better tolerated than $A m B$. ${ }^{[47-49]}$ Furthermore, there is an animal experiment suggesting that the combination of intravenous antifungal drugs with intrathecal administration of LAmB could be beneficial in terms of survival and reduction of fungal load in CSF. ${ }^{[47,50]}$

AmB is easy to combine with human cholesterol cell membrane, ${ }^{[48,49]}$ so the adverse reactions are more and serious. AmB has high toxicity, especially liver and kidney toxicity. Renal toxicity which could lead to lowering glomerular filtration rate and electrolyte disturbances is the most common. Renal function can be restored by early termination of the use of $\mathrm{AmB}^{\left[{ }^{[1]}\right]}$ or replacement of LamB. ${ }^{[11]}$ In addition, some studies $^{[11,52,53]}$ support that preemptive hydration and electrolyte supplementation are the effective methods to minimize the toxicity in middle- and low-income countries (MLICs) ${ }^{[4]}$ Anemia is another common side effect of $A m B,{ }^{[54]}$ the reason is that the effect of the bone marrow on the synthesis of the erythropoietin. ${ }^{[55]}$ 5-FC could get through the BBB easily and has slight adverse reactions, such as gastrointestinal reaction, rash, erythropenia, light degree damage of liver and kidney function, etc. Symptoms can be relieved after stopping taking the drug. The incidence rate of adverse reactions of $F C Z$ is low, the symptoms mainly include gastrointestinal reaction, rash, and so on. Liver and kidney impairment are transient and would returned to normal after drug withdrawal generally. ${ }^{[56]}$

\section{Treatment of high intracranial pressure}

The incidence of HICP in patients with cryptococcal meningitis is more than $50 \% \cdot{ }^{[50]} \mathrm{HICP}$ is the leading cause of death and complications. ${ }^{[4]}$ Therefore, effective control of intracranial pressure for improving clinical symptoms to gain enough time for the success of anti-fungal therapy is of crucial importance. Active treatment of HICP is crucial whether it is HIVassociated patients or not. ${ }^{[57]}$ Methods used to reduce intracranial pressure commonly as follows: ${ }^{[58]}$ (1) Drugs such as mannitol, glycerin fructose, corticosteroids, acetazolamide and so on. While the long-term effect of medical management is not clear that is not used routinely; ${ }^{[57,59]}$ (2) Lumbar puncture. Patients whose intracranial pressure $>2.4 \mathrm{kPa}$ are performed with regular lumbar paracentesis to maintain normal

Table 2: Antifungal therapeutic schedule for $\mathrm{CM}$ patients with renal dysfunction

\begin{tabular}{lll}
\hline Schedule & Course & \\
\hline Induction period & LAmB $3-4 \mathrm{mg} / \mathrm{kg}$ per day $/ \mathrm{ABLC} 5 \mathrm{mg} / \mathrm{kg}$ per day $+5-\mathrm{FC} 100 \mathrm{mg} / \mathrm{kg}$ per day \\
Consolidation period & FCZ $400-800 \mathrm{mg} /$ day or $6-12 \mathrm{mg} / \mathrm{kg}$ per day $\quad \geq 8$ weeks \\
Maintenance period & FCZ $200-400 \mathrm{mg} /$ day or $3-6 \mathrm{mg} / \mathrm{kg}$ per day & $6-12 \mathrm{months}$ \\
\hline
\end{tabular}

CM: cryptococcal meningitis; LAmB: liposome amphotericin B; ABLC: amphotericin B lipid complexes; 5-FC: 5-Flurocytosine; FCZ: fluconazole

Table 3: Antifungal therapeutic schedule for non-HIV associated or non-transplant patients

\begin{tabular}{|c|c|}
\hline Schedule & Course \\
\hline Induction period & $\begin{array}{l}\text { AmB 0.7-1.0 mg/kg per day or LAmB 3-4 mg/kg per day or ABLC } 5 \mathrm{mg} / \mathrm{kg} \text { per day + 5-FC } 100 \mathrm{mg} / \mathrm{kg} \text { per day } \\
4-6 \text { weeks }\end{array}$ \\
\hline $\begin{array}{l}\text { Consolidation period } \\
\text { Maintenance period }\end{array}$ & $\begin{array}{l}\text { FCZ } 400-800 \mathrm{mg} / \mathrm{day} \text { or } 6-12 \mathrm{mg} / \mathrm{kg} \text { per day } \\
\text { FCZ } 200 \mathrm{mg} \text { or } 6 \mathrm{mg} / \mathrm{kg} \text { per day }\end{array}$ \\
\hline
\end{tabular}

HIV: human immunodeficiency virus; AmB: amphotericin B; LAmB: liposome amphotericin B; ABLC: amphotericin B lipid complexes; 5-FC: 5-Flurocytosine; FCZ: fluconazole 
intracranial pressure. Release CSF $10-30 \mathrm{~mL}$ per day until the intracranial pressure has been normalized may be required for a few days. The treatment guideline of IDSA in 2010 points out that this method is the most effective and rapid way to reduce the pressure currently; ${ }^{[24]}$ (3) Lumbar cistern drainage. This method could reduce the number of lumbar puncture and avoid patients' pain. In addition, it is a better method for patients whose intracranial pressure $>3.9 \mathrm{kPa}$ and cannot be controlled effectively by frequent lumbar puncture. Make the open brain pressure fall to $50 \%$ by enough drainage of the cerebrospinal fluid. Regulate the drainage $300-400 \mathrm{~mL}$ per $24 \mathrm{~h}$. It is best no more than 15 days for drainage each time in principle in case of CSF leakage or secondary infection; (4) Ommaya reservoir. This involves a device that allows for ventricle drainage invented by Sheldon and Ommaya ${ }^{[60]}$ in 1963 and applicated as common treatment in adults with cryptococcal meningitis for relief of the symptoms of HICP. The anti-fungal drugs could be injected into ventricles using this device directly and reach effective concentration without influence of BBB. In addition, we could obtain CSF from the ommaya reservoir expediently and securely that it is useful in the evaluation of the state of illness changes and therapeutic effect advantageously. This method reduces the risk of exogenous infection due to the hermetic type structure. However, percutaneous puncture repeatedly may lead to the damage of reservoir or secondary infection; (5) Ventriculoperitoneal shunt. We should consider the ventriculoperitoneal shunt under following circumstances: the control of intracranial pressure is not ideal, recurrent cerebral hernia, occurrence of persistent or progressive cranial nerve defects. Antifungal therapy should be used at the same time to avoid peritoneal cavity infection; (6) Lateral ventricle drainage. If measures above mentioned cannot reduce the intracranial pressure effectively, or there is an expansion of the ventricles, lateral ventricle drainage should be performed in time. But the drainage time should not be too long (2-3 weeks), otherwise it is easy to cause infection. These surgical techniques above could not only reduce intracranial pressure but alsobe used to intrathecal or ventricular injection to improve the therapeutic effect.

\section{Immune therapy}

The main infection routes of Cryptococcus is through the respiratory tract, asymptomatic latent state is the most common infection state. ${ }^{[44]}$ When there is immune function defect in people that could not resist the growth and reproduction of the fungi, Cryptococcus will proliferate and migrate through the blood to other organs in the body, leading to the CM eventually. Therefore, anti-Cryptococcus infection by immune regulation opens up a new way. Anti-fungal drugs combined immunotherapy has been put forward in recent years.

In a phase I clinical study overseas, twenty cases of cryptococcosis are treated with monoclonal antibody from mice aimed at capsule antigen, the result shows that high doses of monoclonal antibodies can reduce the level of polysaccharide antigen in serum temporarily, but there may appear allergic reactions and other side effects. ${ }^{[61]}$

It has been reported that clinical application of interferon in the treatment of fungal resistance in 2004..$^{[62]}$ Jarvis et al. ${ }^{[63]}$ conduct a randomized controlled experiment and show that the rate of fungal clearance is accelerated by adding interferon on the basis of AmB combined with 5-FC, and there is no other side effects. But there is no statistical significance in mortality of patients between group with interferon $100 \mu \mathrm{g}$ (Day 1, Day 3) and group with $100 \mu g$ (Day 1, Day 3, Day 5, Day 8, Day 10, Day 12).

The treatment guidelines set out by Infectious disease society of American in 2010 recommended to give formal antifungal agents combined with IFN- $\gamma$ to patients with persistent infection (whose culture result of CSF is positive after 4 weeks of antifungal therapy with $100 \mu \mathrm{g} / \mathrm{m}^{2}$ for more than $50 \mathrm{~kg}, 50 \mu \mathrm{g} / \mathrm{m}^{2}$ for less than $50 \mathrm{~kg} 3$ times a week for 10 weeks. Small-scale phase 2 clinical trials have shown the good curative effect. However, larger clinical trials are needed to verify.

In recent years, radioimmunotherapy has become an adjuvant immunotherapy for the treatment of Cryptococcus infection. The principle is to use the radioactive substances to label monoclonal antibodies, thus killing the fungi with cytotoxic radiation substance. Bryan et al. ${ }^{[64]}$ have performed animal experiment, demonstrated that RIT is more effective to mice infected with $C$. neoformans than AmB. Besides, RIT could prevent the development of fungal resistance. ${ }^{[42]}$

\section{CONCLUSION}

Higher morbidity and mortality has caused great concern from scholars all over the world. How to make early diagnosis and effective treatment is the key point of the current study. Immunotherapy opens up a new way of treatment for CM. But it is in the bud. Therefore, the development of new drugs with effective antifungal activity and low toxicity as well as effective treatment is still a problem we need to solve.

\section{Financial support and sponsorship} Nil. 


\section{Conflicts of interest \\ There are no conflicts of interest.}

\section{Patient consent \\ No patient involved.}

\section{Ethics approval}

This article does not contain any studies with human participants or animals.

\section{REFERENCES}

1. Park BJ, Wannemuehler KA, Marston BJ, Govender N, Pappas PG, Chiller TM. Estimation of the current global burden of cryptococcal meningitis among persons living with HIV/AIDS. AIDS 2009;23:525-30.

2. Brizendine KD, Pappas PG. Cryptococcal meningitis: current approaches to management in patients with and without AIDS. Curr Infect Dis Rep 2010;12:299-305.

3. Jarvis JN, Bicanic T, Loyse A, Namarika D, Jackson A, Nussbaum JC, Longley N, Muzoora C, Phulusa J, Taseera K, Kanyembe C, Wilson D, Hosseinipour MC, Brouwer AE, Limmathurotsakul D, White N, van der Horst C, Wood R, Meintjes G, Bradley J, Jaffar S, Harrison T. Determinants of mortality in a combined cohort of 501 patients with HIV-associated Cryptococcal meningitis: implications for improving outcomes. Clin Infect Dis 2014;58:736-45.

4. WHO Guidelines Approved by the Guidelines Review Committee. Rapid advice: diagnosis, prevention, and management of cryptococcal disease in HIV-infected adults, adolescents and children. Geneva: World Health Organization; 2011 Dec.

5. Brizendine KD, Baddley JW, Pappas PG. Predictors of mortality and differences in clinical features among patients with cryptococcosis according to immune status. PLoS One 2013;8:e60431.

6. Mirza SA, Phelan M, Rimland D, Graviss E, Hamill R, Brandt ME, Gardner T, Sattah M, de Leon GP, Baughman W, Hajjeh RA. The changing epidemiology of cryptococcosis: an update from populationbased active surveillance in 2 large metropolitan areas, 1992-2000. Clin Infect Dis 2003;36:789-94.

7. Rothe C, Sloan DJ, Goodson P, Chikafa J, Mukaka M, Denis B, Harrison T, van Oosterhout JJ, Heyderman RS, Lalloo DG, Allain T, Feasey NA. A prospective longitudinal study of the clinical outcomes from cryptococcal meningitis following treatment induction with 800 mg oral fluconazole in Blantyre, Malawi. PLoS One 2013;8:e67311.

8. Mwaba P, Mwansa J, Chintu C, Pobee J, Scarborough M, Portsmouth S, Zumla A. Clinical presentation, natural history, and cumulative death rates of 230 adults with primary cryptococcal meningitis in Zambian AIDS patients treated under local conditions. Postgrad Med $J$ 2001;77:769-73.

9. Lesser RL, Simon RM, Leon H, Siegel N. Cryptococcal meningitis and internal ophthalmoplegia. Am J Ophthalmol 1979;87:682-7.

10. Kestelyn P, Taelman H, Bogaerts J, Kagame A, Abdel Aziz M, Batungwanayo J, Stevens AM, Van de Perre P. Ophthalmic manifestations of infections with Cryptococcus neoformans in patients with the acquired immunodeficiency syndrome. Am J Ophthalmol 1993;116:721-7.

11. Sloan DJ, Parris V. Cryptococcal meningitis: epidemiology and therapeutic options. Clin Epidemiol 2014;6:169-82.

12. Kovoor JM, Mahadevan A, Narayan JP, Govindappa SS, Satishchandra P, Taly AV, Shankar SK. Cryptococcal choroid plexitis as a mass lesion: MR imaging and histopathologic correlation. AJNR Am J Neuroradiol 2002;23:273-6.

13. Ueda H, Toribe Y, Kuwae Y, Takeuchi M, Nakayama M, Ida S, Okamoto N, Suzuki Y. An autopsy case of cryptococcal meningoencephalitis: correlation of MRI and pathologic findings. No To Hattatsu 2003;35:499-504. (in Japanese)

14. Loyse A, Wainwright $\mathrm{H}$, Jarvis $\mathrm{JN}$, Bicanic T, Rebe $\mathrm{K}$, Meintjes G, Harrison TS. Histopathology of the arachnoid granulations and brain in HIV-associated cryptococcal meningitis: correlation with cerebrospinal fluid pressure. AIDS 2010;24:405-10.

15. Anekthananon T, Manosuthi W, Chetchotisakd P, Kiertiburanakul S, Supparatpinyo K, Ratanasuwan W, Pappas PG, Filler SG, Kopetskie HA, Nolen TL, Kendrick AS, Larsen RA; BAMSG 3-01 Study Team. Predictors of poor clinical outcome of cryptococcal meningitis in HIV-infected patients. Int J STD AIDS 2011;22:665-70.

16. Megson GM, Stevens DA, Hamilton JR, Denning DW. D-mannitol in cerebrospinal fluid of patients with AIDS and cryptococcal meningitis. J Clin Microbiol 1996;34:218-21.

17. Xu XG, Pan WH, Bi XL, Fang W, Chen M, Zhu Y, Zhou J, Zhou N, Pan B, Li M, Liao WQ, Qi ZT. Comparison of clinical features in patients with persistent and nonpersistent cryptococcal meningitis: twelve years of clinical experience in four centers in China. CNS Neurosci Ther 2013;19:625-31.

18. Hakim JG, Gangaidzo IT, Heyderman RS, Mielke J, Mushangi E, Taziwa A, Robertson VJ, Musvaire P, Mason PR. Impact of HIV infection on meningitis in Harare, Zimbabwe: a prospective study of 406 predominantly adult patients. AIDS 2000;14:1401-7.

19. Moosa MY, Coovadia YM. Cryptococcal meningitis in Durban, South Africa: a comparison of clinical features, laboratory findings, and outcome for human immunodeficiency virus (HIV)-positive and HIVnegative patients. Clin Infect Dis 1997;24:131-4.

20. Makadzange AT, McHugh G. New approaches to the diagnosis and treatment of cryptococcal meningitis. Semin Neurol 2014;34:47-60.

21. Boulware DR, Rolfes MA, Rajasingham R, von Hohenberg M, Qin Z, Taseera K, Schutz C, Kwizera R, Butler EK, Meintjes G, Muzoora C, Bischof JC, Meya DB. Multisite validation of cryptococcal antigen lateral flow assay and quantification by laser thermal contrast. Emerg Infect Dis 2014;20:45-53.

22. Kisenge PR, Hawkins AT, Maro VP, McHele JP, Swai NS, Mueller A, Houpt ER. Low CD4 count plus coma predicts cryptococcal meningitis in Tanzania. BMC Infect Dis 2007;7:39.

23. Abassi M, Boulware DR, Rhein J. Cryptococcal meningitis: diagnosis and management update. Curr Trop Med Rep 2015;2:90-9.

24. Perfect JR, Dismukes WE, Dromer F, Goldman DL, Graybill JR, Hamill RJ, Harrison TS, Larsen RA, Lortholary O, Nguyen MH, Pappas PG, Powderly WG, Singh N, Sobel JD, Sorrell TC. Clinical practice guidelines for the management of cryptococcal disease: 2010 update by the infectious diseases society of america. Clin Infect Dis 2010;50:291-322.

25. Fan XH, Feng GD, Yang YN, Dai W, Zhao G. Diagnostic value of May-Grunwald-Giemsa staining of cerebrospinal fluid in patients with cryptococcal meningitis. J Int Neurol Neurosurg 2013;40:221-2.

26. He JY, He HY, Meng ZH, Jin BF. Disscussion of early diagnosis and curative effect of cryptococcal meningitis (30 cases report). Zhongguo Shen Jing Jing Shen Ji Bing Za Zhi 2007;33:433-5.

27. Rhein J, Boulware DR. Prognosis and management of cryptococcal meningitis in patients with human immunodeficiency virus infection. Neurobehav HIV Med 2012;4:45-61.

28. Jarvis JN, Lawn SD, Vogt M, Bangani N, Wood R, Harrison TS Screening for cryptococcal antigenemia in patients accessing an antiretroviral treatment program in South Africa. Clin Infect Dis 2009;48:856-62.

29. Longley N, Jarvis JN, Meintjes G, Boulle A, Cross A, Kelly N, Govender NP, Bekker LG, Wood R, Harrison TS. Cryptococcal antigen screening in patients initiating ART in South Africa: a prospective cohort study. Clin Infect Dis 2016;62:581-7.

30. Temstet A, Roux P, Poirot JL, Ronin O, Dromer F. Evaluation of a 
monoclonal antibody-based latex agglutination test for diagnosis of cryptococcosis: comparison with two tests using polyclonal antibodies. J Clin Microbiol 1992;30:2544-50.

31. Perfect JR. Fungal diagnosis: how do we do it and can we do better? Curr Med Res Opin 2013;29 Suppl 4:3-11.

32. Wang LX, Shi LB, Chen WS, Xiao ZH, Cheng D. Detection of capsular polysaccharide antigen by latex agglutination test for early diagnosis of meningitis or pneumonia caused by Cryptococcus neoformans. Jian Yan Yi Xue 2008;23:55-7.

33. Bennett JE, Bailey JW. Control for rheumatoid factor in the latex test for cryptococcosis. Am J Clin Pathol 1971;56:360-5.

34. Tanner DC, Weinstein MP, Fedorciw B, Joho KL, Thorpe JJ, Reller L. Comparison of commercial kits for detection of cryptococcal antigen. J Clin Microbiol 1994;32:1680-4.

35. Gade W, Hinnefeld SW, Babcock LS, Gilligan P, Kelly W, Wait K, Greer D, Pinilla M, Kaplan RL. Comparison of the PREMIER cryptococcal antigen enzyme immunoassay and the latex agglutination assay for detection of cryptococcal antigens. J Clin Microbiol 1991;29:1616-9.

36. Lawn SD, Wood R. Point-of-care urine antigen screening tests for tuberculosis and cryptococcosis: potential for mortality reduction in antiretroviral treatment programs in Africa. Clin Infect Dis 2012;54:739-40.

37. Sanchini A, Smith IM, Sedlacek L, Schwarz R, Tintelnot K, Rickerts V. Molecular typing of clinical Cryptococcus neoformans isolates collected in Germany from 2004 to 2010. Med Microbiol Immunol 2014;203:333-40

38. Sow D, Tine RC, Sylla K, Djiba M, Ndour CT, Dieng T, Ndiaye JL, Faye B, Ndiaye D, Gaye O, Dieng Y. Cryptococcal meningitis in Senegal: epidemiology, laboratory findings, therapeutic and outcome of cases diagnosed from 2004 to 2011. Mycopathologia 2013;176:443-9.

39. Yao ZW, Lu X, Shen C, Lin DF. Comparison of flucytosine andfluconazole combined with amphotericin B for the treatment of HIV-associated cryptococcal meningitis: a systematic review and meta-analysis. Eur J Clin Microbiol Infect Dis 2014;33:1339-44.

40. Day JN, Chau TT, Lalloo DG. Combination antifungal therapy for cryptococcal meningitis. N Engl J Med 2013;368:2522-3.

41. Saag MS, Cloud GA, Graybill JR, Sobel JD, Tuazon CU, Johnson PC, Fessel WJ, Moskovitz BL, Wiesinger B, Cosmatos D, Riser L, Thomas C, Hafner R, Dismukes WE. A comparison of itraconazole versus fluconazole as maintenance therapy for AIDS-associated cryptococcal meningitis. National Institute of Allergy and Infectious Diseases Mycoses Study Group. Clin Infect Dis 1999;28:291-6.

42. Gullo FP, Rossi SA, Sardi Jde C, Teodoro VL, Mendes-Giannini MJ, Fusco-Almeida AM. Cryptococcosis: epidemiology, fungal resistance, and new alternatives for treatment. Eur J Clin Microbiol Infect Dis 2013;32:1377-91.

43. Saag MS, Graybill RJ, Larsen RA, Pappas PG, Perfect JR, Powderly WG, Sobel JD, Dismukes WE. Practice guidelines for the management of cryptococcal disease. Infectious Diseases Society of America. Clin Infect Dis 2000;30:710-8.

44. Dromer F, Bernede-Bauduin C, Guillemot D, Lortholary O; French Cryptococcosis Study Group. Major role for amphotericin B-flucytosine combination in severe cryptococcosis. PLoS One 2008;3:e2870.

45. Fang W, Fa Z, Liao W. Epidemiology of Cryptococcus and cryptococcosis in China. Fungal Genet Biol 2015;78:7-15.

46. Hodge $\mathrm{G}$, Cohen $\mathrm{SH}$, Thompson GR 3rd. In vitro interactions between amphotericin B and hydrocortisone: potential implications for intrathecal therapy. Med Mycol 2015;53:749-53.

47. Gazzoni AF, Capilla J, Mayayo E, Guarro J. Efficacy of intrathecal administration of liposomal amphotericin B combined with voriconazole in amurine model of cryptococcal meningitis. Int $J$ Antimicrob Agents 2012;39:223-7.

48. Jarvis JN, Dromer F, Harrison TS, Lortholary O. Managing cryptococcosis in the immunocompromised host. Curr Opin Infect Dis 2008;21:596-603.

49. Sloan D, Dlamini S, Paul N, Dedicoat M. Treatment of acute cryptococcal meningitis in HIV infected adults, with an emphasis on resource-limited settings. Cochrane Database Syst Rev 2008;4:CD005647.

50. Capilla J, Flavia A, Mayayo E, Guarro J. Efficacy of intrathecal liposomal amphotericin B plus oral posaconazole in the treatment of acute meningeal cryptococcosis in a murine model. Int $J$ Antimicrob Agents 2013;42:282-3.

51. Bicanic T, Wood R, Meintjes G, Rebe K, Brouwer A, Loyse A, Bekker LG, Jaffar S, Harrison T. High-dose amphotericin B with flucytosine for the treatment of cryptococcal meningitis in HIV-infected patients: a randomized trial. Clin Infect Dis 2008;47:123-30.

52. Kambugu A, Meya DB, Rhein J, O’Brien M, Janoff EN, Ronald AR, Kamya MR, Mayanja-Kizza H, Sande MA, Bohjanen PR, Boulware DR. Outcomes of cryptococcal meningitis in Uganda before and after the availability of highly active antiretroviral therapy. Clin Infect Dis 2008;46:1694-701.

53. Pappas PG, Chetchotisakd P, Larsen RA, Manosuthi W, Morris MI, Anekthananon T, Sungkanuparph S, Supparatpinyo K, Nolen TL, Zimmer LO, Kendrick AS, Johnson P, Sobel JD, Filler SG. A phase II randomized trial of amphotericin B alone or combined with fluconazole in the treatment of HIV-associated cryptococcal meningitis. Clin Infect Dis 2009;48:1775-83.

54. Miller RP, Bates JH. Amphotericin B toxicity. A follow-up report of 53 patients. Ann Intern Med 1969;71:1089-95.

55. Catalán M, Montejo JC. Systemic antifungals. Pharmacodynamics and pharmacokinetics. Rev Iberoam Micol 2006;23:39-49. (in Spanish)

56. $\mathrm{Xu} \mathrm{N}$, Wen $\mathrm{H}$, Chen $\mathrm{JH}$. Recent development of drug therapy in cryptococcal meningitis. Zhongguo Zhen Jun Xue Za Zhi 2006;1:248-52.

57. Brizendine KD, Pappas PG. Cryptococcal meningitis: current approaches to management in patients with and without AIDS. Curr Infect Dis Rep 2010;12:299-305.

58. Liliang PC, Liang CL, Chang WN, Lu K, Lu CH. Use of ventriculoperitoneal shunts to treat uncontrollable intracranial hypertension in patients who have cryptococcal meningitis without hydrocephalus. Clin Infect Dis 2002;34:E64-8.

59. Graybill JR, Sobel J, Saag M, van Der Horst C, Powderly W, Cloud G, Riser L, Hamill R, Dismukes W. Diagnosis and management of increased intracranial pressure in patients with AIDS and cryptococcal meningitis. The NIAID Mycoses Study Group and AIDS Cooperative Treatment Groups. Clin Infect Dis 2000;30:47-54.

60. Sheldon P, Ommaya AK. Ventricular dilatation masking the presence of cerebral tumors. Acta Radiol Diagn (Stockh) 1963;1:628-37.

61. Larsen RA, Pappas PG, Perfect J, Aberg JA, Casadevall A, Cloud GA, James R, Filler S, Dismukes WE. Phase I evaluation of the safety and pharmacokinetics of murine-derived anticryptococcal antibody $18 \mathrm{~B} 7$ in subjects with treated cryptococcal meningitis. Antimicrob Agents Chemother 2005;49:952-8.

62. Netea MG, Brouwer AE, Hoogendoorn EH, Van der Meer JW Koolen M, Verweij PE, Kullberg BJ. Two patients with cryptococcal meningitis and idiopathic CD4 lymphopenia: defective cytokine production and reversal by recombinant interferon- gamma therapy. Clin Infect Dis 2004;39:e83-7.

63. Jarvis JN, Meintjes G, Rebe K, Williams GN, Bicanic T, Williams A, Schutz C, Bekker LG, Wood R, Harrison TS. Adjunctive interferon- $\gamma$ immunotherapy for the treatment of HIV-associated cryptococcal meningitis: a randomized controlled trial. AIDS 2012;26:1105-13.

64. Bryan RA, Jiang Z, Howell RC, Morgenstern A, Bruchertseifer F, Casadevall A, Dadachova E. Radioimmunotherapy is more effective than antifungal treatment in experimental cryptococcal infection. $J$ Infect Dis 2010;202:633-7. 